\title{
Multiple Spatial-Frequency Tuning of Electrical Responses from Human Visual Cortex*
}

\author{
C. W. Tyler, P. Apkarian, and K. Nakayama
}

Smith-Kettlewell Institute of Visual Sciences, 2232 Webster Street, San Francisco, CA 94115, U.S.A.

\begin{abstract}
Summary. Human occipital potentials evoked by stimulation with a counterphase flickering grating were recorded by a digital narrowband filter technique. The data showed a surprising degree of narrow tuning to particular spatial frequencies in addition to the expected narrow temporal frequency tuning. At each temporal frequency, there could be two or more peaks of response to different spatial frequencies, each distinct from the whole field flicker response. Variations in this multiple spatial frequency tuning were investigated as a function of luminance, electrode location, and temporal frequency for several observers. The results are interpreted in terms of many stimulus-specific resonant neural circuits within the brain, and suggest that it is possible to make a highly detailed exploration of the responses of neural circuits to visual stimulation.
\end{abstract}

Key words: VEP - Pattern - Spatial frequency - Cortex.

It is often tacitly assumed that the electrical activity of the brain recorded from the scalp should reflect the properties of the visual system as inferred from psychophysics. This approach is not necessarily the most fruitful, because when a correspondence is obtained, it provides little new information about the visual system that was not already known from the psychophysics. On the other hand, new discoveries, dissimilar from psychophysics, are frequently considered to show some kind of artifact of the electrical activity which is ignored in the description of visual function.

We propose to take a rather different approach which may prove more incisive in understanding the electrical activity of the brain, and the visual

\footnotetext{
* Supported by NIH Grants No. 5 R01 EY01582, No. 1 R01 EY02124, NIH General Research Grant No. 5 S01 RR05566 and The Smith-Kettlewell Eye Research Foundation
} 

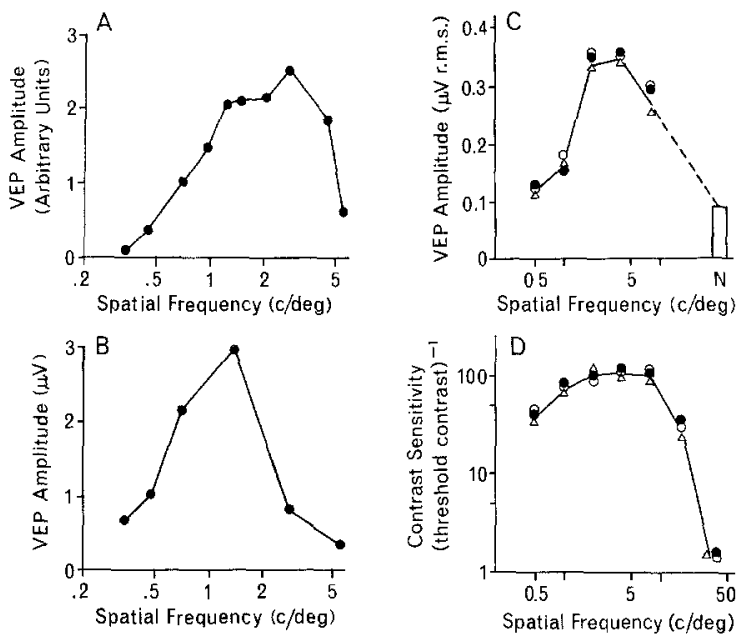

Fig. 1. Examples of spatial frequency tuning in previous VEP studies. Transient pattern response amplitudes from Fig. 8 of Rietveld et al. (1967) are replotted in $\mathbf{A}$. In B, data from Harter and White (1970) from their Fig. 5 show tuning in the 90-110 ms negative component of the transient evoked response. Freeman and Thibos (1975) found narrow spatial frequency tuning of the steady-state evoked potential (C) relative to the psychophysical contrast sensitivity (D) obtained on the same observer. For these latter data, linear VEP amplitude and log psychophysical sensitivity should be equivalent because of the log linear contrast relationship (see Fig. 5)

system in particular. This approach is to study the stimulus determinants of evoked potentials in fine detail without forcing them into a mould formed by psychophysical data. Correspondences to previous information are followed up as a secondary activity, both in the direction of human psychophysics and equally in the direction of animal neurophysiology. At this initial stage, our investigation is largely descriptive, delineating the phenomena to be discovered by a detailed examination of stimulus determinants.

As a relevant example of information in the electrical responses which has been disregarded because of a mismatch to psychophysics, consider the tuning of visual evoked potentials (VEP) as a function of bar width (or its inverse, spatial frequency) in a grating stimulus. This kind of study has been conducted in sufficient detail to obtain an estimate of the spatial frequency tuning of responses by Rietveld et al. (1967), Harter and White (1970), Armington et al. (1971) and Freeman and Thibos (1975). Typical examples of the responses obtained are depicted in Fig. 1, together with a psychophysical sensitivity function from Freeman and Thibos (1975) for comparison. None of these authors mentioned the narrowness of the VEP tuning or compared it with the relatively broad psychophysical function. Yet the VEP seems to be consistently tuned to as little as 2 octaves at half-height, whereas the psychophysical function spreads over at least 4 octaves at half contrast.

The narrow tuning of the VEP is a surprise, since it means that there are gratings of some spatial frequencies which are highly visible and yet produce little response from the cortex under the recording conditions used. Rather than 
allow this mismatch to be detrimental to the understanding of evoked potentials, we decided to explore the question of narrow tuning in the VEP, to see if it was a robust phenomenon. We have studied the detailed characteristics of the tuning to ensure that it was not a result of some other effect, such as an accelerating contrast function, a non-linear response to the edges of the bars in the grating, or electrical subtraction between responses from different cortical areas.

\section{Methods}

Stimulus

Stimulus patterns were presented on the face of a Hewlett-Packard cathode ray tube (CRT) display (Model 1332A, P31 Phosphor) following a modification of the method employed by Schade (1956) and Campbell and Green (1965). Either a flickering homogeneous field or a counterphase flickering grating of varying stimulus dimensions could be generated.

Linearity of the intensity modulation was determined by plotting luminance as a function of $Z$-axis voltage. A selenium photocell used in all light measures, including those of contrast, was operated in the photovoltaic mode. Long-term stability of the photocell output was periodically tested against a $100 \mathrm{ft} 1$. Gamma Scientific (Model 220) standard source. The mean luminance of the $10 \times 12 \mathrm{~cm}$ display screen was set at the midpoint of the measured linear operating range; this setting allowed for contrast modulation of up to 0.80 . To increase or decrease the mean luminance, appropriate neutral density filters were utilized. Contrast, which was measured according to the standard Michelson (1927) definition, could be adjusted in 0.1 log unit steps.

\section{Recording and Data Analysis}

Steadystate visually evoked potentials were recorded with a bipolar electrode placement based on the optimum position for pattern-evoked potentials determined by many previous authors (Harter and White, 1970; Cobb et al., 1967; Jeffreys and Axford, 1972). Unless stated otherwise, the recording electrodes were placed $3 \mathrm{~cm}$ above the inion and $3 \mathrm{~cm}$ above and lateral; the ear served as ground.

To improve the signal-to-noise ratio of the steady state evoked potential, the EEG was analyzed by means of a synchronous narrow-band filtering technique (see Fig. 2) basically similar to those employed by Van der Tweel et al. (1958) and Fricker (1962). The amplified EEG signals were first pre-filtered through a Kron-hite (Model 330-M) $24 \mathrm{db} /$ octave bandpass filter with cutoff frequencies set at $0.1 \mathrm{log}$ unit below and above the stimulus alternation rate, eliminating harmonics of the fundamental response frequency. The pre-filtered signals were then passed through a synchronous constant narrow bandwidth $(0.3 \mathrm{~Hz})$ commutating filter, the center frequency of which was equal to and driven by the second harmonic of the stimulus frequency.

Cortical potentials were analyzed at twice the stimulating frequency because we have found, as have several authors (e.g., Spekreijse, 1966; Cobb et al., 1967), that the major frequency component of the evoked potentials produced is equal to the phase alternation or stimulus reversal rate rather than the fundamental stimulus frequency which is $1 / 2$ the phase alternation rate. To distinguish between the stimulus frequency and the stimulus alternation rate, cycles per second (Hz) are used to denote the former while reversals per second (rps) are used to denote the latter.

The filter output was full-wave rectified and integrated over a 10 -s recording epoch with the results displayed on a digital counter.

\section{Noise Analyses}

Unlike most previous authors using a synchronous filter technique, we felt it important always to distinguish carefully the neural activity evoked by the stimulus from the uncorrelated background 


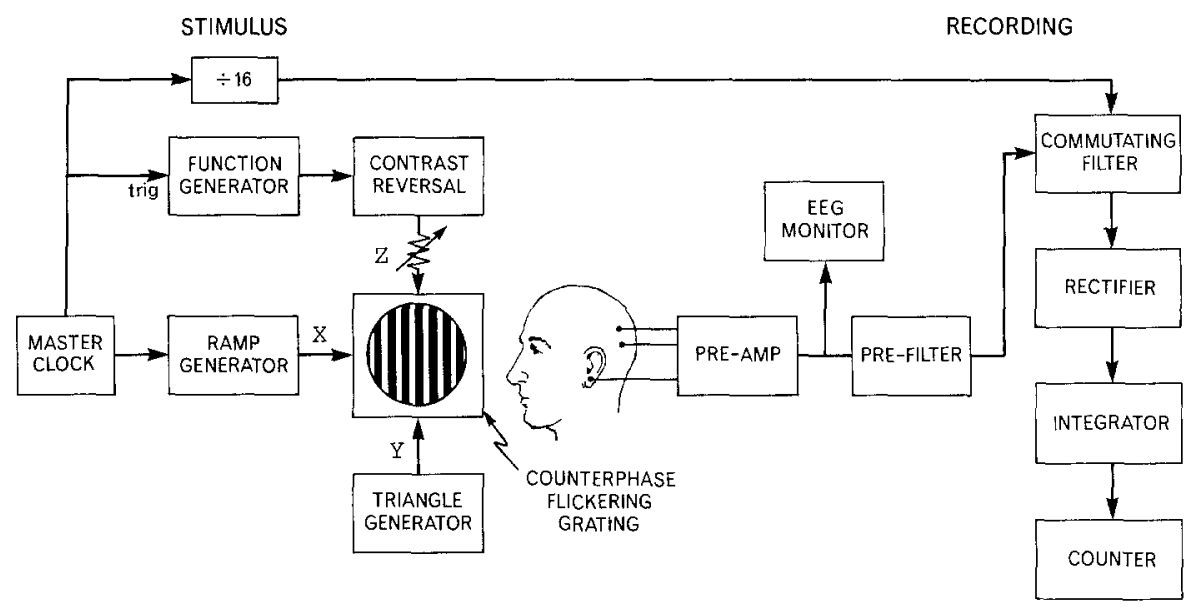

Fig. 2. Block diagram of apparatus for narrowband filtering of VEP using a sinusoidal grating alternating in counterphase at any desired temporal frequency

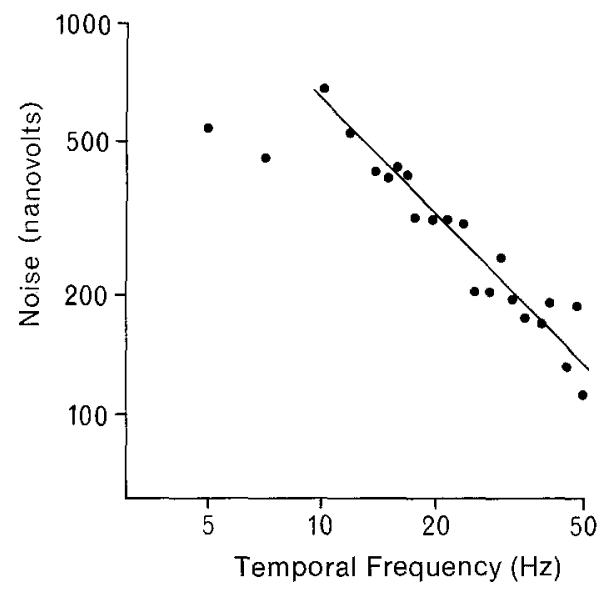

Fig. 3. Level of physiological noise as a function of temporal frequency in logarithmic coordinates. (The bandwidth of the analysis is constant in linear coordinates, as is usual for Fourier analysis). These data, which conform to a slope of -1 over the range $10-50 \mathrm{rps}$, correspond to the Fourier spectrum of the resting EEG

activity at the stimulus frequency. The evoked potential amplitudes are therefore expressed in signal-to-noise ratios. The average level of the noise (uncorrelated background activity) is obtained by recording the response to a homogeneous stationary field at random intervals during each recording session. The ratio of the mean signal amplitude for each stimulus condition to this noise amplitude is then taken as the response measure.

The average physiological noise level as a function of temporal frequency $(10$ to $50 \mathrm{~Hz})$ is plotted for observer CWT in Fig. 3. Careful measurements of possible noise artifact originating from the equipment (e. g., multistage amplifier noise) indicate that the electronic system noise contributes a negligible proportion to the physiological noise response. An extensive degree of up to a log unit attenuation of the physiological noise response can occur within the $2+$ octave range of temporal frequencies tested. Similar noise amplitude and frequency data have previously been described (Spekreijse, 1966). The noise data presented here have been fitted by inspection over most of the frequency range with a theoretical filter function which has a slope of -1 . Since the data can show noise levels as low as $30 \mathrm{nV}$ at high temporal frequencies, the signal-to-noise ratio approach allows measurement of extremely low signal amplitudes. 
One concern with plotting the signal in relation to the noise is that local fluctuations in noise level as a function of temporal frequency would appear as changes in the signal. However, their resultant contribution can be empirically determined and have been found under our recording conditions to exhibit no sudden amplitude fluctuations across the whole temporal frequency range. The smooth attenuation observed in our noise data indicates the narrow peaks in the VEP spatiotemporal specificity, which are presented in the Results section, are not contaminated by spurious noise fluctuations or attenuation. By expressing the evoked potential amplitudes in signal-to-noise ratios, comparisons across temporal frequency can be made in terms that take account of the sources of attenuation between the signal generation site and the electrode. Since the resultant attenuation is of the order of 1 decade, it is clearly of great importance to compensate for it.

\section{Procedure}

The observers, resting comfortably in a supine position, viewed an overhead mirror image of the 10 $\times 12^{\circ} \mathrm{CRT}$ display which appeared at a distance of $57 \mathrm{~cm}$. They were instructed to fixate a $20^{\prime}$ black fixation spot in the center of the screen.

Background EEG activity was monitored continuously to ensure observer vigilence during each recording epoch. The slightest EEG distortion, muscle artifact, or observer disturbance was cause for cancellation of the trial and additional readings were then obtained. Recording was continued only when the normal and appropriate baseline noise level was attained.

Spatial-frequency tuning curves across the temporal-frequency spectrum were obtained by the random presentation of spatial frequencies ranging from approximately 0.2 to 20 cycles per degree; this procedure was repeated for each temporal frequency (10 to $50 \mathrm{rps})$, the order of which was also randomized. Temporal tuning curves for uniform field responses and contrast functions were obtained in a similar fashion, i.e., by randomizing the appropriate variables.

\section{Results}

\section{Spatial Tuning and Effects of Luminance}

As an initial study of the spatialfrequency tuning, we selected a temporal modulation frequency of $16 \mathrm{rps}(8 \mathrm{~Hz})$ and measured the $16 \mathrm{~Hz}$ response as a function of spatial frequency over a range of mean luminance levels. The first surprise was that not only is the response narrowly tuned in spatial frequency, but there is more than one peak in the function (Fig. 4). The double-peaked function is quite reliable, as it appears at all four of the luminance levels which show a significant response to the stimulus. Furthermore, we have found it is reproducible from day to day and year to year.

There are strong effects of luminance on the response, even though the basic double-peaked function is not disrupted. The clearest effect is the lack of response below $0.1 \mathrm{~cd} / \mathrm{m}^{2}$, which approximates the photopic threshold. However, above this level the response appears to remain nearly constant over a two log unit range rather than increasing in proportion to luminance or absolute modulation amplitude. In this respect, the VEP again departs radically from psychophysics, since psychophysical sensitivity varies roughly in direct proportion to absolute modulation amplitude at, say, $5 \mathrm{c} / \mathrm{deg}$ and $8 \mathrm{~Hz}$ in the luminance range from 0.1 to $10 \mathrm{~cd} / \mathrm{m}^{2}$ (deLange, 1957; Van Nes, 1968).

However, there is one aspect of the luminance responses in which the data do behave as expected. There is an orderly progression of the frequencies of peak response, each decreasing by about one octave as luminance is reduced (compare Van Nes, 1968). 


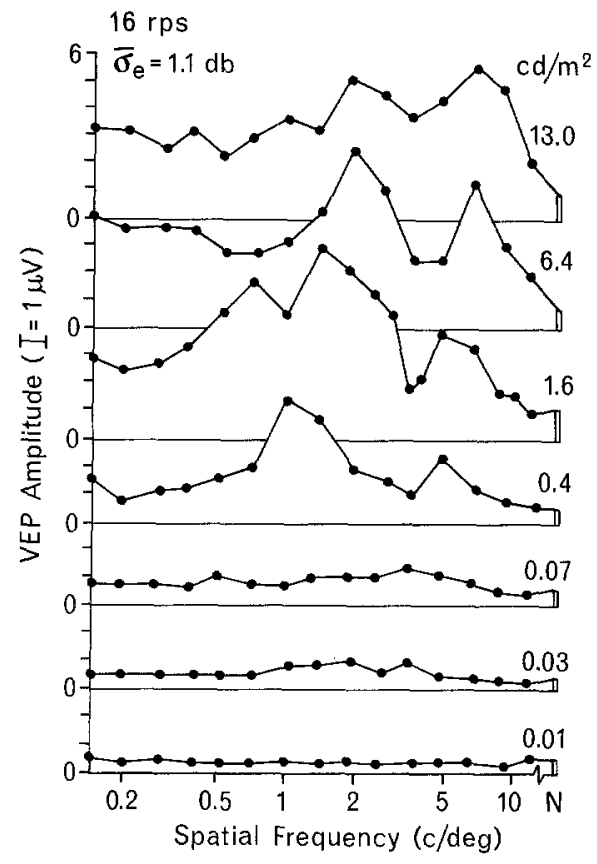

Fig. 4. Spatial tuning of VEP at $16 \mathrm{rps}$ as a function of mean luminance of the gratings. Note the reliable occurrence of a double-peaked function with very narrow spatial frequency tuning. The mean $\log$ standard error $\left(\bar{\sigma}_{\mathrm{e}}\right)$ in averaged over all the data $(1.0 \mathrm{db} \approx 10 \%)$

\section{Estimation of Bandwidth of the Peaks}

The data of Fig. 4 show multiple peaks which are evidently tuned much more narrowly than suspected from the data in the literature (Fig. 1). In estimating the spatialfrequency bandwidth of the response peaks, it is important to know the linearity of the contrast function at each peak. If the response showed an accelerating amplitude as contrast was increased, a peak measured in terms of response amplitude might appear narrower than its true tuning in terms of input sensitivity of the cortex.

Accordingly, we measured the contrast function in the region of the two peaks of interest. These are plotted in terms of VEP amplitude as a function of log contrast, following Campbell and Maffei (1970) (Fig. 5). The contrast functions are fairly linear in these coordinates, particularly for the high spatial frequency peak at $7 \mathrm{c} / \mathrm{deg}$, which is the narrower of the two. A linear contrast function implies that such narrow spatialfrequency tuning can be obtained without distortion of the tuning curve by contrast non-linearities.

If plotted in linear coordinates, both functions would be linear or tend to decelerate rather than accelerate, so that any distortion of the tuning curve would be towards broadening as opposed to narrowing of the bandwidth.

An estimate of the bandwidth of the response peaks in Fig. 3 may be obtained by two methods. To remain strictly with the data obtained, we may measure the bandwidth of the response at half-amplitude above baseline noise response. Alternatively, for direct comparison with psychophysical and neurophysiological estimates, we may measure the bandwidth at half contrast. 
Fig. 5. VEP as a function of contrast at the spatial frequencies of the two peaks in Fig. 4. Both contrast functions are fairly linear, and therefore contribute little to the narrowness of the spatial frequency tuning, particularly in the case of the high spatial frequency peak (see text)
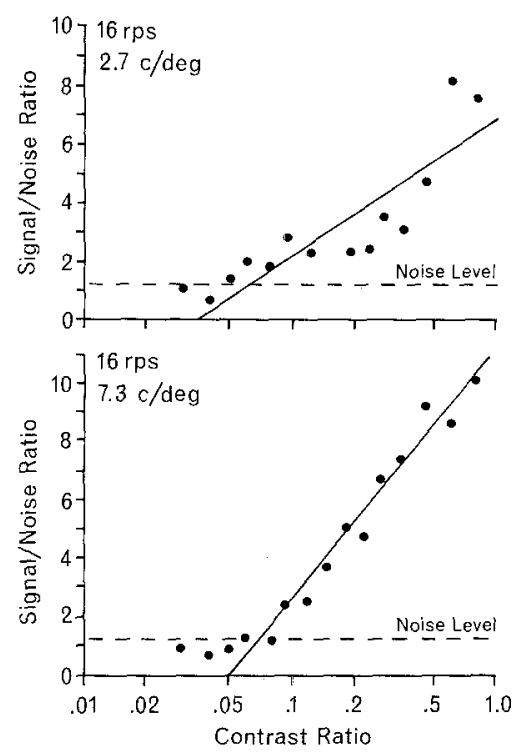

For this method the bandwidth is measured at the response amplitude produced by stimulation at half contrast (i.e., as found in the contrast function of Fig. 5). This half-contrast method avoids contamination by a nonlinearity of the contrast function or a response threshold, and should therefore be considered a more stringent criterion.

Using the half-height method, the full bandwidths of the two peaks in Fig. 3 are 1.1 octaves at $7 \mathrm{c} / \mathrm{deg}$ and 1.5 octaves at $2 \mathrm{c} / \mathrm{deg}$. On the other hand, the more stringent half-contrast method based on the contrast functions of Fig. 5 gives bandwidths as narrow as 0.5 octave at $7 \mathrm{c} / \mathrm{deg}$ and an unchanged 1.5 octaves at $2 \mathrm{c} / \mathrm{deg}$. By the standards of either psychophysics or neurophysiology these represent very narrow tuning. It is too early to assess the physiological significance of these results, but our first concern was to determine the range of conditions under which such narrow tuning would be obtained.

\section{Spatiotemporal Frequency Map}

We therefore measured spatialfrequency tuning as a function of temporal frequency for four observers (CWT, DS, SA, BC). The data are shown in isometric projection in Fig. 6A-D. Note the use of the signal/noise ratio plot as described in the Methods section. These projections illustrate a number of features of the spatiotemporal frequency (STF) characteristics of the steady state evoked potential, and one is replotted as a contour map in Fig. 7 (see below).

1. Temporal frequency tuning can be very narrow and can depend on spatial frequency. A large peak at one spatial frequency may be completely altered by a 
A.

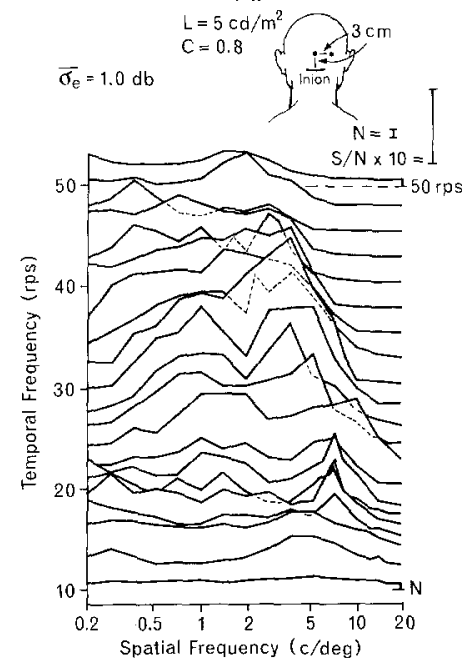

c.

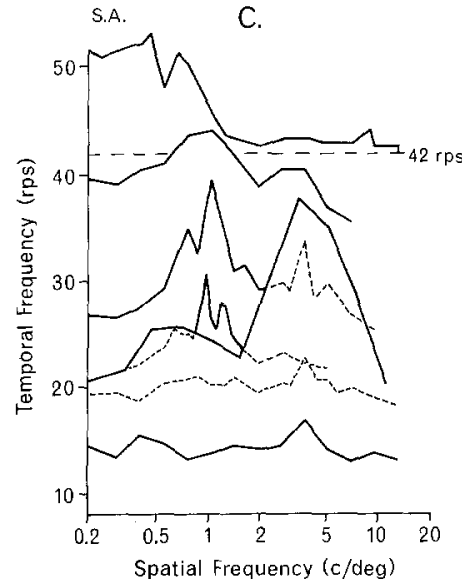

B.

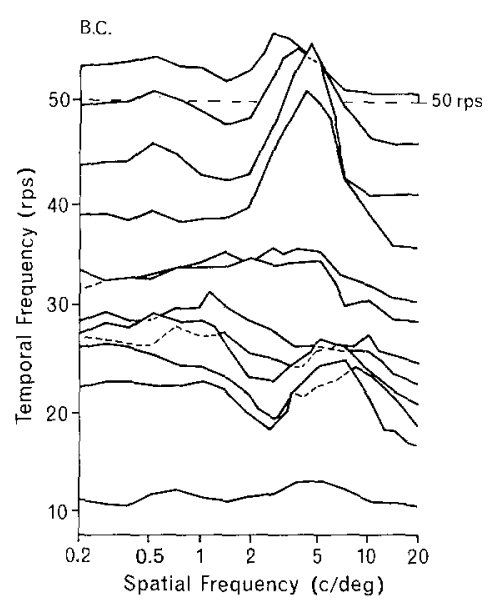

D.

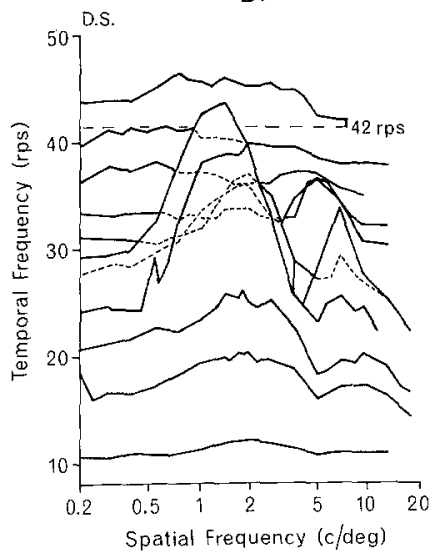

Fig. 6. Spatial frequency tuning as a function of temporal frequency (rps) for four different observers (A-D). Each curve is plotted from a baseline at its frequency specified on the ordinate. Most are down to the amplitude of the noise (shown as $\mathrm{N}$ in inset) above the baseline by $20 \mathrm{c} / \mathrm{deg}$ (as depicted for $50 \mathrm{rps}$.). Note high degree of stimulus specificity in spatial and temporal frequency of response. Although the detail differs for each observer at the same electrode position, multiple narrow spatial frequency tuning is prevalent

temporal frequency change of only $10 \%$. For example, in Fig. 7 , note that as temporal frequency is varied from 30 to $32.5 \mathrm{rps}$, there is a $50 \%$ drop from the peak response. Such narrow tuning implies that a great deal of information about the spatial characteristics of the evoked potential is lost if only one temporal frequency is used for analysis, as has been characteristic of past studies (e.g., Campbell and Maffei, 1970). Narrow temporal-frequency tuning is well known in studies of uniform field flicker (Van der Tweel, 1964; Spekreijse, 


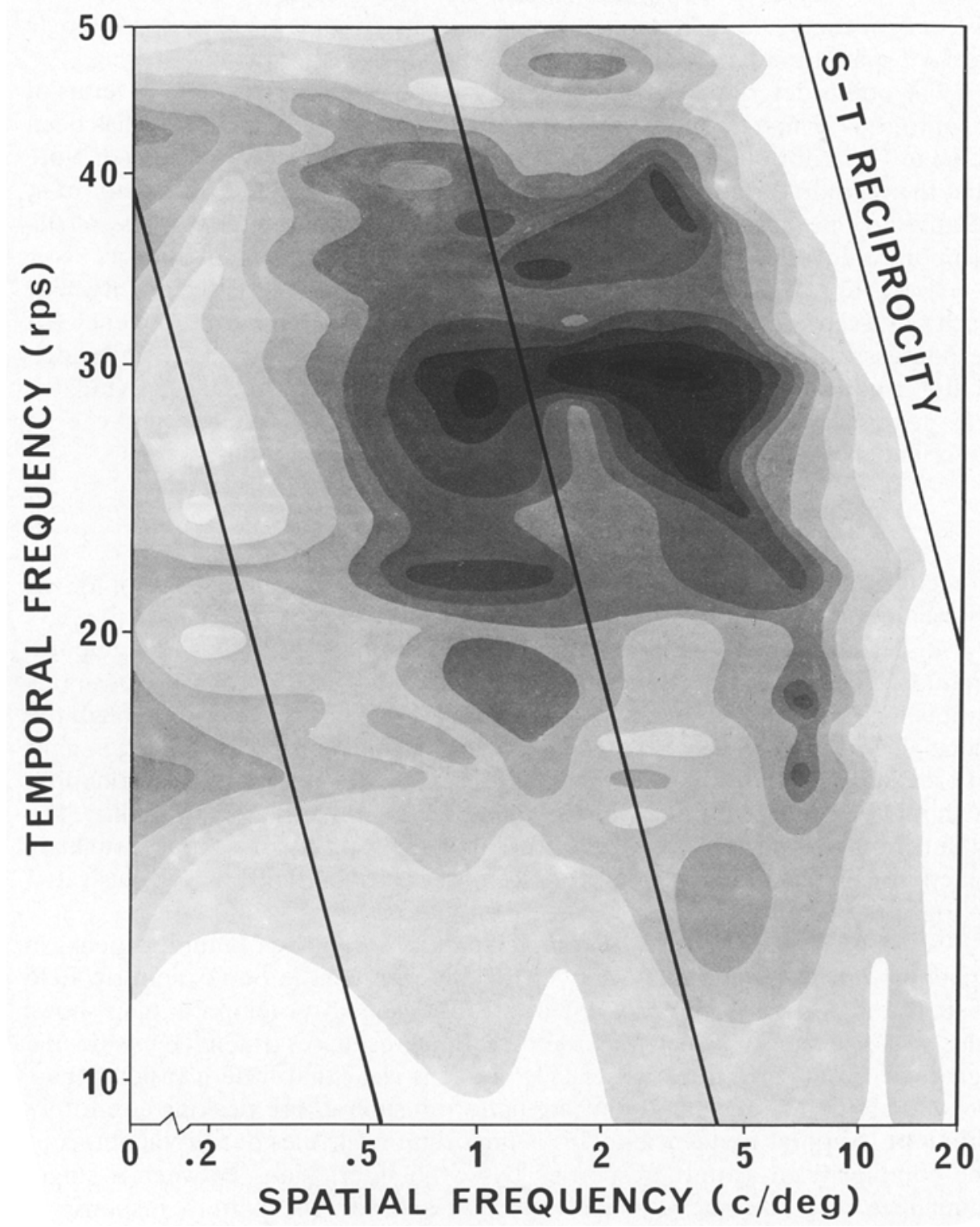

Fig. 7. Contour map of the spatial frequency tuning of Fig. 6A. Note that the temporal frequency ordinate is expanded by a factor of 4 relative to the abscissa, so the oblique lines represent strict spatiotemporal reciprocity. Zero spatial frequency represents the uniform field

1966; Regan, 1966, 1968), but temporal-frequency tuning of pattern response has previously been thought to be rather broad and exhibit no narrow peaks (Regan, 1972).

2. Spatialfrequency tuning is often as narrow as \pm 0.6 octave at half-amplitude throughout the range investigated, bearing out the generality of 
the narrow tuning described in a previous section. In some cases there is a single peak of spatialfrequency sensitivity at a given temporal frequency.

The points described here are summarized by replotting the data in terms of a contour STF map to obtain an overview of the multiple tuning. This has been done in Fig. 7 for observer CWT, on whom the most detail was obtained. Note that the temporal frequency axis (ordinate) has been expanded by a factor of 4 , relative to the spatial frequency axis (abscissa), so that the narrowness of the temporal frequency tuning is therefore even more marked than it appears.

3. All observers show a tendency toward spatiotemporal reciprocity. For each observer, there is a pronounced tendency for high temporal frequency $\left(f_{t}\right)$ responses to be limited to the lower spatial frequency $\left(f_{s}\right)$ region, while high spatial frequency responses occur only at lower temporal frequencies (Fig. 7). This reciprocity is most marked at high spatiotemporal frequency, and can be described as an absence of response when

$$
f_{s} \cdot f_{t}>300 \text { r.c/s.deg, }
$$

a reciprocity which is reminiscent of that seen in threshold psychophysics, especially for high temporal frequencies (Van Nes, 1968).

4. Multiple peak responses occur at different spatial and temporal frequencies for different observers. The detailed localization of peak positions differs in both spatial and temporal frequency across observers, even though the same electrode placement was always used. This finding is not surprising in light of previous work, most of which also reports extensive inter-observer variability. It should be noted, however, that in spite of this inter-observer variability, the within observer variability is very low, and replications of spatial frequency functions can be obtained from the same observer over months, as is illustrated in Fig. $10 \mathrm{~A}$.

5. A final observation is that complex pattern responses of multiple peaks in spatialfrequency tuning can occur in regions where little or no uniform field response is evident (e.g., for CWT at $28 \mathrm{rps}$ ). The spatiotemporal map shows nicely the extent to which the uniform field responses (at left) invade the territory of the pattern responses (Fig. 7). It is clear that little invasion occurs beyond about $0.5 \mathrm{c} / \mathrm{deg}$, and that the pattern responses are peaking at entirely different temporal frequencies. This is important as it rules out explanations of the complexity of pattern responses based on interactions between a single luminance response and a single pattern response. The pattern response is complex in itself, presumably made up of a number of different pattern responses, each tuned to a different spatial frequency and summing in some manner to produce the observed responses.

\section{Electrode Location}

Evoked potential studies over the past 20 years have accentuated the importance of electrode location on the form of the transient evoked potential. While we have not attempted a comprehensive study at this stage, it seemed 
Fig. 8. Spatial frequency tuning for observer $\mathrm{BC}$ at a slightly different electrode configuration $(3 \mathrm{~cm}$ and $6 \mathrm{~cm}$ above the inion). Note the radical differences compared with Fig. 6B

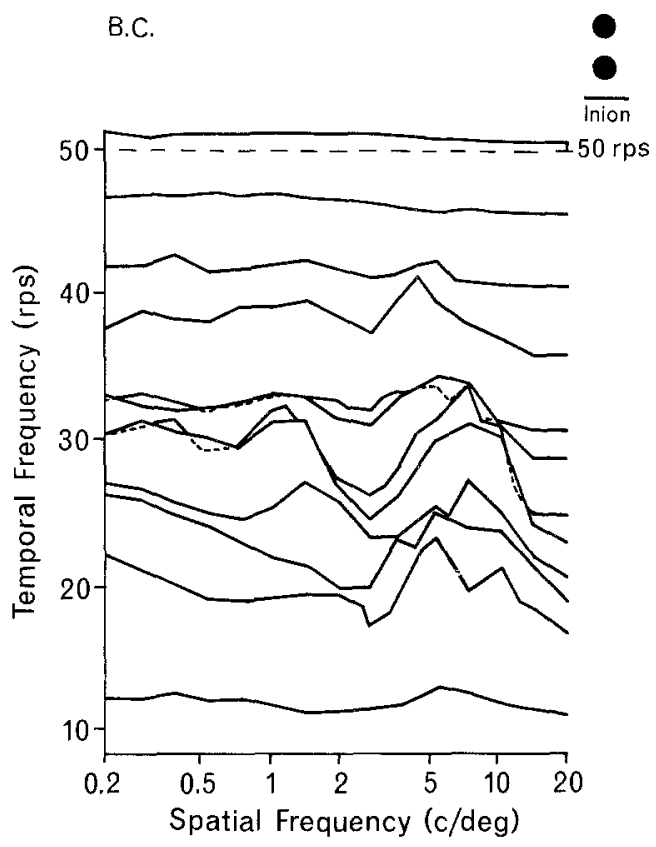

apposite to establish whether nearby electrodes would have similar or radically different spatiotemporal frequency response characteristics.

We therefore recorded a set of STF tunings for two observers with the active electrode $3 \mathrm{~cm}$ vertically above the reference electrode on the midline, rather than $3 \mathrm{~cm}$ horizontally to the right. The complete STF map for these new electrode positions is shown in isometric projection for observer BC (Fig. 8). Examples for a few temporal frequencies are selected for direct comparison with the original horizontal placement (Fig. 9).

It is immediately obvious that a small $(4.2 \mathrm{~cm})$ shift in electrode position can have a radical effect on the STF specificity of the steady-state evoked response. Although in many cases the spatial frequency tuning is quite similar for the two electrode placements (Fig. 8), different peaks often appear, and a healthy response at one placement may be essentially absent at the other (Fig. 9).

Nevertheless, the general characteristics of the response are similar at the new electrode position. The features of narrow spatial frequency tuning, ultranarrow temporal frequency tuning and spatiotemporal reciprocity are all present. It is only in the particular details that the responses differ.

\section{Are the Peaks Due to Electrical Summation Artifacts?}

Another familiar issue in VEP recording is the possible occurrence of response reduction or cancellation at the recording electrode, by electrical subtraction of responses in different temporal phases due to passive volume conduction 


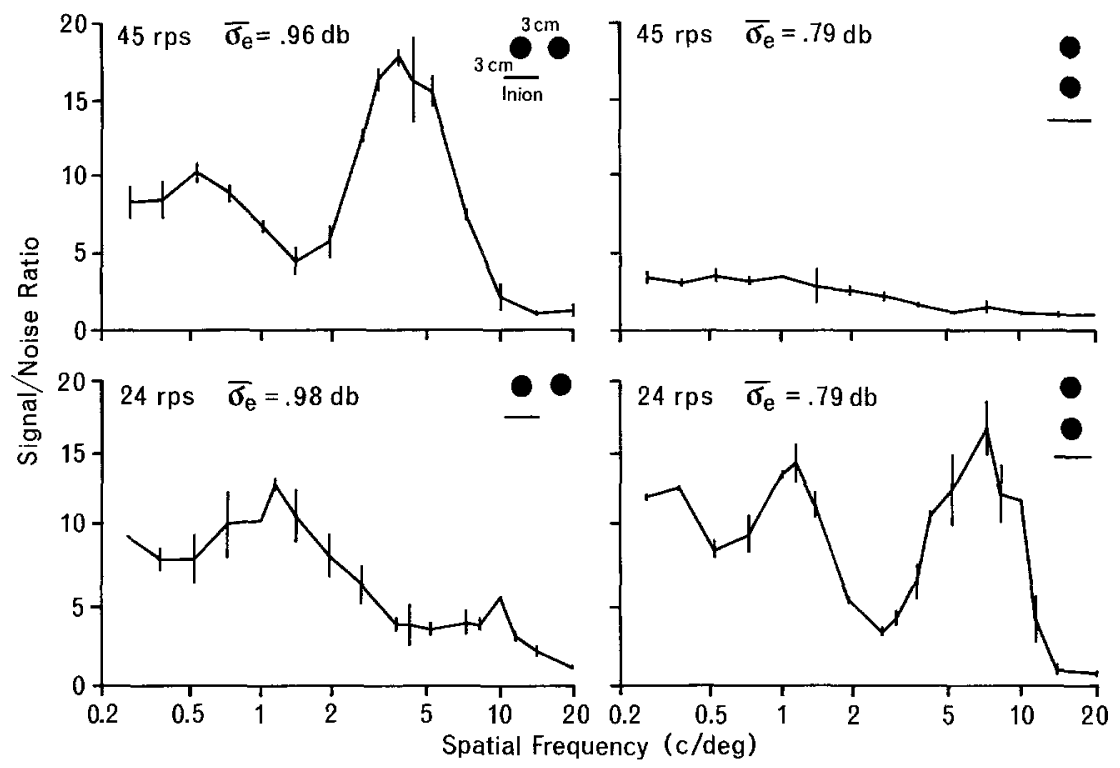

Fig. 9. Direct comparison of effect of horizontal versus vertical electrode placement at two temporal frequencies

emanating from different neural populations. One relevant example is that responses of opposing polarity may be generated from stimulation of the upper and lower parts of the field. This has been demonstrated both for transient VEP (Michael and Halliday, 1970; Jeffreys and Axford, 1972) and for steady-state VEP (Vigotti, 1973). In order to ensure that such effects were not producing our basic results, we repeated the spatialfrequency tuning measurements with two additional conditions. The field of stimulation was restricted to the lower left quadrant, to eliminate both upper/lower, and left/right field interactions. In addition, the temporal phase of the responses was determined for each reading by inspection of the digital filter output at the end of each recording. This allowed a specification of the temporal phase to within $1 / 16$ of a cycle $\left(22.5^{\circ}\right)$, which was sufficiently accurate for the present purposes.

For this control one observer was still available to us (CWT). We chose to measure the response in the region where the best response had been obtained ( $28 \mathrm{rps}$ ), but with only one quadrant of stimulation. Even though there was a lapse of one year between the two recordings, the response amplitude profile (Fig. 10A, upper panel) is extremely similar to the full field response (see Fig. $6 \mathrm{~A}$ ), showing a replication of the double-peaked function with peaks at about 1.0 and $4.0 \mathrm{c} / \mathrm{deg}$ with a trough amplitude of about half the $4 \mathrm{c} / \mathrm{deg}$ peak amplitude in each case. This double-peaked function therefore cannot be the result of any kind of passive electrical summation between upper and lower, or left and right field representations in the cortex.

Despite this demonstration, there remains the possibility that the curious spatialfrequency profile of the responses results from subtractive electrical 

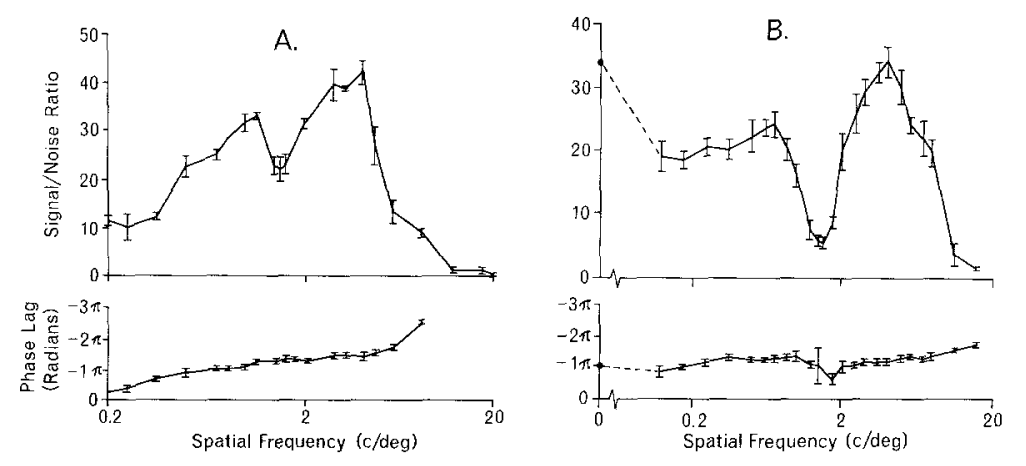

Fig. 10. A Amplitude and phase plots of response as a function of spatial frequency, at 28 rps with stimulation of lower left quadrant of visual field for observer CWT. Upper panel shows amplitude response, which is a good replication of the corresponding full field curve in Fig. 6A. Lower panel shows phase lag as a function of spatial frequency. Note that this is not a Bode plot of temporal frequency and no theoretical from of the temporal phase response is implied by the amplitude distribution over spatial frequency. B Amplitude and phase plots as in A., but for observer DL at 24 rps with full field stimulation

interactions between different neural populations which are both stimulated even with the restricted quadrant stimulation. If such interactions are by electrical subtraction at the scalp electrode site, they would constitute a recording artifact which would not be a reflection of neural responses. On the other hand, if they can be shown not to be due to an electrical artifact, they must represent a neural effect of some kind carrying information about brain function. By recording the temporal phase of the response, we are able to show that electrical subtraction is not occurring in this case, and that the double-peaked nature of the function is therefore produced by some as yet undetermined type of neural mechanism.

In particular, the variation in temporal phase of the response with spatial frequency is shown in Fig. 10A, lower panel. Several points should be made from these data. First, consider the question of phase subtraction in the trough of the response. If two mechanisms or neural populations have the same amplitudes and different temporal phases to the same stimulus, the summed response is actually greater than that of the largest of the two responses until the phase difference exceeds $\pm 120^{\circ}$. The phase plot of Fig. $10 \mathrm{~A}$ shows that the phase difference between the two regions of peak response is at maximum $90^{\circ}$, which should produce phase addition rather than subtraction between the two mechanisms. Thus the presence of a dip between the two peaks must have a neural basis.

To further substantiate this case, we include amplitude and phase data with fullfield stimulation for another observer (DL), who exhibits almost complete loss of response at a spatial frequency of about $2 \mathrm{c} / \mathrm{deg}$ (although the stimulus was strongly visible to the observer). In this case the phase difference between the regions of peak response is virtually zero, although the phase was difficult to determine in the low response region (Fig. 10B). Electrical subtraction is ruled 
out by the absence of a measured temporal phase shift, even though such phase shifts can be due to numerous factors, including the geometry of the neural population, as well as differences in latency.

\section{Discussion}

The results of the steady-state evoked potential exploration suggest an unprecedented degree of specificity and tuning as a function of both spatial and temporal frequency. This tuning is not amenable to explanation by phase cancellation of responses from different cortical locations or subpopulations.

Most noteworthy and surprising is the fact that evoked potential amplitude can vary abruptly with changes in spatial frequency, a result having little in common with the broad psychophysical function. In fact, the narrowness of the peaks is more similar to the tuning curves obtained from single neurons in the cat and primate cortex (Campbell et al., 1969; Maffei and Fiorentini, 1973; Schiller et al., 1976) or the tuning curves of spatiallytuned channels as inferred from adaptation studies (Blakemore and Campbell, 1969). What needs to be answered is why evoked potential recording, a technique which appears to sample from a large heterogeneous population of neurons, is so specifically tuned?

The most interesting possibility is that the narrow spatial and temporal tuning seen in the scalp response is due to the specificity of neural connections in the region of cortex processing the stimulus. It is likely that for a homogeneous region of retina, each neuron or neural circuit responding best to a certain size of stimulus, will have a similar synaptic organization (or one of a few types of synaptic organization, such as simple and complex types of cell (Hubel and Wiesel, 1962). Each type of synaptic organization will have specific temporal characteristics, which may well include resonant features. Such resonances may arise either from feedback connections within each neural circuit, or from facilitation arising through simultaneous stimulation of aggregates of similar types of neural circuit across the cortex.

Given this view of the underlying neurophysiology, it is reasonable to expect the degree of spatial and temporal frequency specificity which we report here. For example, neural circuits organized for spatial stimuli of $5 \mathrm{c} / \mathrm{deg}$ may happen to contain a temporal resonance at $40 \mathrm{rps}$. Changing the spatial frequency to 10 $\mathrm{c} / \mathrm{deg}$ will presumably no longer stimulate those circuits, but the circuits appropriate for the $10 \mathrm{c} / \mathrm{deg}$ grating may resonate at some other temporal frequency, or may have some change in the nature of the dendritic fields that abolishes the resonant characteristics altogether. Thus, the steady-state response will depend on the precise arrangement of excitation and inhibition, including synaptic delays and neural path lengths, and hence show selective tuning under those conditions which are optimal for resonance.

This interpretation, although plausible, is intended merely as a conceptual framework within which to consider the results. Other interpretations may be possible, which we are in the process of testing. However, this conception gives 
the hope that by detailed analysis in the domain that we have explored, it may be possible to determine the processing characteristics of closely specified sets of neural circuits within the human visual system.

\section{References}

Armington, J.C., Corwin, T.R., Marsetta, R.: Simultaneously recorded retinal and cortical responses to patterned stimuli. J Opt Soc Am 61, 1514-1521 (1971)

Blakemore, C., Campbell, F.W.: On the existence of neurones in the human visual system selectively sensitive to the orientation and size of retinal images. Physiol (Lond) 203, 237-260 (1969)

Campbell, F.W., Cooper, G.F., Enroth-Cugell, C.: The spatial selectivity of the visual cells of the cat. J Physiol (Lond) 203, 223-235 (1969)

Campbell, F.W., Green, D.G.: Optical and retinal factors affecting visual resolution. J Physiol (Lond) 181, 576-593 (1965)

Campbell, F.W., Maffei, L.: Electrophysiological evidence for the existence of orientation and size detectors in the human visual system. J Physiol (Lond) 207, 635-652 (1970)

Cobb, W.A., Morton, H.B., Ettlinger, G.: Cerebral potentials evoked by pattern reversal and their suppression in visual rivalry. Nature 216, 1123-1125 (1967)

Freeman, R.D., Thibos, L.N.: Visual evoked responses in humans with abnormal visual experience. J Physiol (Lond) 247, 711-724 (1975)

Fricker, S.J.: Narrow-band filter techniques for the detection and measurement of evoked responses. Electroencephalogr Clin Neurophysiol 14, 411-413 (1962)

Harter, M.R., White, C.T.: Evoked cortical responses to checkerboard patterns: Effect of check-size as a function of visual acuity. Electroencephalogr Clin Neurophysiol 28, 48-54 (1970)

Hubel, D.H., Wiesel, T.N.: Receptive fields, binocular interaction and functional architecture in the cat's visual cortex. J Physiol (Lond) 160, 106-154 (1962)

Jeffreys, D.A., Axford, J.G.: Source locations of pattern-specific components of human visual evoked potentials. I. Component of striate cortical origin. Exp Brain Res 16, 1-21 (1972)

Lange, $H$. de: Attenuation characteristics and phase-shift characteristics of the human fovea-cortex systems in relation to flicker-fusion phenomena. Technical University, Delft, Thesis. The Hague: Junk 1957

Maffei, L., Fiorentini, A.: The visual cortex as a spatial frequency analyzer. Vision Res 13, 1255-1267 (1973)

Michelson, A.A.: Studies in Optics. Chicago: University of Chicago Press 1927

Perry, N.W., Jr., Childers, D.G.: The Human Evoked Response. Springfield: Thomas 1969

Regan, D.: Some characteristics of average steady-state and transient responses evoked by modulated light. Electroencephalogr Clin Neurophysiol 20, 238-248 (1966)

Regan, D.: A high frequency mechanism which underlies visual evoked potentials. Electroencephalogr Clin Neurophysiol 25, 231-237 (1968)

Regan, D.: Evoked Potentials in Psychology, Sensory Physiology and Clinical Medicine. London: Chapman and Hall 1972

Reitveld, W.J., Tordoir, W.E.M., Hagenouw, J.R.B., Lubbers, J.A., Spoor, Th.A.C.: Visual evoked responses to blank and to checkerboard patterned flashes. Acta Physiol Pharmacol Neurol 14, $259-285$ (1967)

Schade, O.H.: Optical and photoelectric analog of the eye. J Opt Soc Am 46, 721-739 (1956)

Schiller, P.H., Finlay, B.L., Volman, S.F.: Quantitative studies of single-cell properties in monkey striate cortex. III. Spatial frequency. J Neurophysiol 39, 1334-1351 (1976)

Stevens, C.F.: Quantitative theories in neurophysiology, Chapter 10. In: Neurophysiology. A Primer. New York: Wiley 1966

Spekreijse, H.: Analysis of EEG responses in man. Thesis, University of Amsterdam. The Hague: Junk 1966

Van der Tweel, L.H.: Relation between psychophysics and electrophysiology of flicker. Doc Ophthalmol 18, 287-304 (1964) 
Van der Tweel, L.H., Sem-Jacobsen, C.W., Kamp, A., Van Leeuwen, W.S., Veringa, F.T.H.: Objective determination of response to modulated light. Acta Physiol Pharmacol Neurol 7, 528-529 (1958)

Van Nes, F.L.: Experimental studies in spatiotemporal contrast transfer by human eye. Thesis, University of Utrecht. The Hague: Junk 1968

Vigotti, M.: Potenziali evocati dalla parte superiore o inferiore dell compo visivo. Fondazione Giorgio Ronchi, Atti 28, 187-189 (1973)

Received January 20, 1978 